\title{
Short Report of a Preliminary Open Study of Synofit-Containing Bio-Curcumin, Greenlipped Mussel and Blackcurrant Leaf Extract in Arthritis
}

\author{
Jiangang $Q u^{1}$, Christian Mélot ${ }^{2}$, Thierry Appelboom ${ }^{1 *}$ \\ ${ }^{1}$ Department of Rheumatology, Molière-Longchamp Hospital, Brussels, Belgium \\ ${ }^{2}$ Department of Emergency, Erasme University Hospital, Brussels, Belgium \\ Email: *thierry.appelboom@erasme.ulb.ac.be
}

Received 20 September 2015; accepted 30 October 2015; published 2 November 2015

Copyright (C) 2015 by authors and Scientific Research Publishing Inc.

This work is licensed under the Creative Commons Attribution International License (CC BY).

http://creativecommons.org/licenses/by/4.0/

(c) () Op Open Access

\section{Abstract}

To evaluate the potential benefit of Synofit-an association of Curcumin, Perna canaliculus greenmussels and blackcurrant leaf extracts, a real life open study was performed among 86 adult out patients suffering from Fibromyalgia $(n=22)$, low back pain $(n=33)$ or knee osteoarthritis $(n=$ 31) who accepted to take 3 tablets a day during 1 week then 2 capsules of Synofit during 2 months in addition to their conventional therapy (mainly analgesics and anti-inflammatory) and then to report their evaluation of this complementary treatment. Statistical analysis included paired $t$ test and when possible Wilcoxon signed rank test. Accordingly, the intermediate analysis showed that already within 4 weeks of treatment, an improvement quoted as "light" was statistically reported in patients with low back pain and knee osteoarthritis but not among those with fibromyalgia on pain, physical condition, global assessment of a benefit, quality of life but not on joint stiffness (although joint stiffness considered for the whole group was statistically improved). The limited number of patients and time duration of the study and the absence of double blind controlled study do not allow concluding on the efficacy but these preliminary analyses obtained from an intermediate analysis are encouraging for further studies.

\section{Keywords}

Synofit, Greenlipped Mussels, Curcumin, Blackcurrant, Arthritis

\footnotetext{
*Corresponding author.
} 


\section{Introduction}

Nutritional agents with a favorable safety profile generate an interest for their potential benefit especially in conditions when no cure exists or, as in osteoarthritis when most therapies focus on pain relief or rely on therapies associated with substantial gastrointestinal, renal and cardio-vascular effect [1].

Among patients consulting a physician for rheumatic with rhematic complain, among the most frequent diagnoses, fibromyalgia, low back pain, and knee osteoarthritis are diagnosed; their prevalence in the U.S. population is respectively evaluated in 2005 to 5.0 million, 59 million and 27 million [2].

A high number of these patients look for complementary and alternative medicine to gain symptomatic relief and avoid iatrogenic illnesses.

Potential therapeutic effects of Curcumin have been stressed in the prevention and treatment of various inflammatory chronic diseases, and especially in primary knee osteoarthritis [3] [4].

The observation that Maoris are high consumers of green lipped mussels (Perna canaliculus) has contributed to the development of derived extracts to be included in preparation as an anti-arthritic product. Data from a systematic review of the nutritional supplement Perna canaliculus as an adjunctive treatment to conventional medication suggest that the extract may be superior to placebo and can exist as a credible alternative in mild or moderate osteoarthritis [5].

Perna canaliculus is a source of omega 3 long chain polyinsaturated fatty acids gaining its composition by filter feeding microorganisms from sea water, estimated to 1000 times more than other fish oils [6].

Yet, the benefit of Liprinol (a New Zealand green lipped mussel preparation) is considered as small [7].

Recently a new formulation of an association of three components potentially effective in osteoarthritis has been introduced as a new complementary medicine in Belgium and the benefit of this association named Synofit is evaluated in this preliminary study in the real life of patients suffering from fibromyalgia, low back pain and knee osteoarthritis.

The intermediate analyses after 4 - 8 weeks of treatment are presented here. Synofit is a liquid formula with main active ingredients pure 100\% greenlipped mussel (Perna canaliculus), bio-curcumine and blackcurrant leaf extract.

\section{Methods}

Synofit 3 capsules a day during 1 week, then 2 capsules daily have been prescribed as supplement to 86 adults out patients (23 males and 63 females) consulting their rheumatologist for chronic pain (more than 3 months), altering their quality of life condition despite regular anti-inflammatory and analgesic therapy; pain was associated to fibromyalgia $(n=22)$, lumbar $(n=33)$ or knee osteoarthritis $(n=31)$; the follow up visit was performed after 4 and if possible 8 weeks.

Efficacy parameters included pain, physical condition, joint stiffness; all these parameters were estimated by a 0 - $100 \mathrm{~mm}$ visual analogue score.

In addition, the evaluation included a global assessment by the patient of the benefit of the Synofit $(-1=$ worse; 0 = unchanged; +1 = slightly better; +2 = better; $+3=$ much better), and finally an evaluation of the improvement on their quality of life $(-1=$ worse; $0=$ unchanged; $+1=$ slightly better; $+2=$ better; $+3=$ much better).

The statistical analysis was performed by an independent statistician and included a paired test and when possible a Wilcoxon signed rank test.

\section{Results}

The preliminary analysis includes the data collected from the follow up of 33/86 patients.

\subsection{Statistical Analysis of the Whole Group}

Data are indicated in Table 1 and can be summarized as follows; after a mean follow up of $4.6 \pm 2.4$ weeks, we observed a slight but significant reduction of pain, an improvement of the physical condition and of articular stiffness; patients felt significantly "slightly better" and which was confirmed by a positive global assessment of the efficacy and of the quality of life by the patients.

Age, sex and treatment duration did not seem to influence the response to therapy (Chi ${ }^{2}$ test, $\mathrm{p}=0.2049$ ). 
Table 1. Whole group.

\begin{tabular}{cllc}
\hline & Before & After & p value \\
\hline Number of patients & $\mathrm{n}=86$ & $\mathrm{n}=33$ & \\
Age & $60 \pm 13$ & \\
Male/female & $23 / 63$ & $4.6 \pm 2.4$ & 0.0001 \\
Duration of Synofit ${ }^{\circledR}$ administration (weeks) & & $4.9 \pm 2.7$ & 0.0001 \\
Pain, VAS (0 - 10) & $6.6 \pm 1.7$ & $4.6 \pm 2.7$ & 0.0157 \\
Physical condition, VAS (0 - 10) & $6.2 \pm 1.8$ & $4.3 \pm 3.1$ & $1.0 \pm 0.24$ \\
Joint stiffness, VAS $(0-10)$ & $5.6 \pm 2.6$ & $0.85 \pm 1.12$ & \\
Evaluation of the benefit $(-1$ to +3$)$ & & & \\
Effect on the quality of life $(-1$ to +3$)$ & &
\end{tabular}

Results are expressed as mean $\pm \mathrm{SD}$, paired t test.

\subsection{Analysis of Separated Groups}

The data are indicated in Table 2.

In the group of Fibromyalgia patients, Synofit did not bring any significant benefit neither on the 5 parameters; pain, physical condition, articular stiffness, nor on the global assessment of efficacy and of quality of life.

In contrast, patients with low back pain experienced a statistically significant reduction of pain and an improvement of their physical condition, but joint stiffness remained unchanged. Globally, they felt better and appreciated the benefit associated to the administration of Synofit.

Similarily, among patients with knee osteoarthritis, pain and physical conditions slightly improved; and despite the lack of benefit on joint stiffness, patients appreciated the administration of Synofit and expressed a slighly but significant improvement on their quality of life.

\section{Discussion}

This study did not evaluate the potential benefit of separately Curcumin, Perna canaliculus or Blackcurrant leaf extract but of their association which so far has not been explored.

The preliminary data of the intermediate analysis can be summarized as follows; the administration of this association named Synofit, during about 1 month improved the clinical condition of patients suffering from low back pain and knee osteoarthritis but not of those with fibromyalgia; the benefit was slight but significant.

In the group of responders, the benefit was reported on the severity of pain, the physical condition, the quality of life, but not on joint stiffness; and satisfaction was expressed by the patients. This effect was significant but remained modest.

Pain, reduced mobility and reduced quality of life are the most frequent complains of osteoarthritis and thses parametres seem to be improved by the association of the 3 extracts.

The interest of this experience is limited because the number of patients are very limited, the study is not controlled and the duration is 1 month only.

The follow up only included 33 out of the 86 patients; the absence of follow up in a high proportion of patients (53) can be explained by the lack of response or by the disappearance of the symptoms; yet since the time of the intermediate analysis, 8 additional patients have been seen for follow up, have reported a benefit (but their data could not be included in this analysis).

The benefit was qualified as "slightly better"; this can be explained by the short duration (1 month) of the administration; interestingly a similar assessment was reported by the 8 additional patients who took Synofit for a longer period.

It is not excluded that longer administration of Synofit can be beneficial for treating such patients.

A pure placebo effect can explain also the "slight" benefit of the therapy but then how to explain the absence of response on all the parameters in fibromyalgia patients in general more sensitive to placebo effect and especially during the first months of drug administration. 
Table 2. Each groups.

\begin{tabular}{|c|c|c|c|}
\hline Fibromyalgia $(n=22)$ & Before & After & $\mathrm{p}$ value \\
\hline Pain, VAS $(0$ - 10) & $7.9 \pm 1.1$ & $7.8 \pm 1.3$ & 0.178 \\
\hline Physical condition, VAS (0 - 10) & $7.5 \pm 1.4$ & $7.8 \pm 1.3$ & 0.178 \\
\hline Joint stiffness, VAS (0 - 10) & $7.7 \pm 1.3$ & $8.3 \pm 0.96$ & 0.182 \\
\hline Evaluation of the benefit $(-1$ to +3$)$ & & $0.17 \pm 0.41$ & \\
\hline Effect on the quality of life $(-1$ to +3$)$ & & $0.17 \pm 0.41$ & \\
\hline Low back pain $(\mathrm{n}=33)$ & Before & After & $\mathrm{p}$ value \\
\hline Pain, VAS $(0$ - 10) & $6.1 \pm 1.5$ & $4.3 \pm 2.7$ & 0.0008 \\
\hline Physical condition, VAS (0 - 10) & $5.8 \pm 1.6$ & $3.8 \pm 2.6$ & 0.0012 \\
\hline Joint stiffness, VAS (0 - 10) & $4.9 \pm 2.5$ & $3.9 \pm 2.9$ & 0.164 \\
\hline Evaluation of the benefit $(-1$ to +3$)$ & & $1.24 \pm 1.20$ & \\
\hline Effect on the quality of life $(-1$ to +3$)$ & & $1.13 \pm 1.15$ & \\
\hline Knee osteoarthritis $(\mathrm{n}=31)$ & Before & After & $\mathrm{p}$ value \\
\hline Pain, VAS $(0$ - 10) & $6.2 \pm 1.7$ & $4.5 \pm 2.5$ & 0.034 \\
\hline Physical condition, VAS $(0-10)$ & $5.8 \pm 2.0$ & $4.1 \pm 2.4$ & 0.0339 \\
\hline Joint stiffness, VAS (0 - 10) & $4.8 \pm 2.5$ & $3.3 \pm 3.3$ & 0.0651 \\
\hline Evaluation of the benefit $(-1$ to +3$)$ & & $1.08 \pm 1.44$ & \\
\hline Effect on the quality of life $(-1$ to +3$)$ & & $0.82 \pm 1.25$ & \\
\hline
\end{tabular}

Results are expressed as mean $\pm \mathrm{SD}$, paired t test.

By the way, we cannot exclude that the concomittant therapies influenced our data.

One can question the limited relevance of the clinical parameters used in this study; they reflect the real life and except for joint stiffness, the benefit was similarily reported for the parameters.

Interestingly, the benefit was observed in low back pain and knee osteoarthritis but not in fibromyalgia; the rapidity of the effect (within 1 month) can be explained by a anti-inflammatory effect rather than by a pure analgesic effect or a cartilage repairing effect; experimental evidences exist that Perna canaliculus extract exerts an anti-inflammatory effect [7]; similarily, Curcumin, the other major constituent of Synofit has been demonstrated as efficient on pain and function [8].

In experimental models, both reduce the production of inflammation mediators such as TNF alpha, Interferon gamma [7]... Other studies have suggested that the Canaliculus extract was capable to modify the microbiota profile and notably reduce Clostridium presence [9]. Synofit also contains small amounts of Blackcurrant leaf rich in anti-oxidant phenols and used as complementary medicine in the management of rheumatic pain.

A recent study has shown that the supplementation of green lipped mussel oil blend significantly attenuated skeletal muscle slow troponin I and TNF alpha after muscle damaging exercise as well as delayed onset muscle soreness, suggesting that the benefit could also be due to a muscle resistance effect [10].

\section{Conclusion}

In conclusion, the interpretation of this intermediate analysis is difficult; yet, Curcumin, greenlipped mussels and blackcurrant leaf have the reputation to bring some benefit in arthritis; for the first time, they are associated in one capsule and within 4 weeks of administration in low back pain and knee osteoarthritis, a slight but significant benefit is observed; the data are encouraging and support the further development of clinical trials. 


\section{References}

[1] Leong, D.J., Choudhury, M., Hirsh, D., Hardin, J., Cobelle, N. and Sun, H. (2013) Neutraceuticals: Potential for Chondroprotection and Molecular Targeting of Osteoarthritis. International Journal of Molecular Sciences, 14, 2306323085. http://dx.doi.org/10.3390/ijms141123063

[2] Lawnence, R., Felson, D., Helmid, C., et al. (2008) Estimates of the Prevalence of Arthritis and Other Rheumatic Conditions in the United States. Arthritis \& Rheumatology, 58, 26-35. http://dx.doi.org/10.1002/art.23176

[3] Aggarwal, B. and Harikumar, K. (2009) Potential Therapeutic Effects of Curcumin, the Anti-Inflammatory Agent against Neurodegenerative, Cardiovascular, Pulmonary, Metabolic, Auto-Immune and Neoplastic Diseases. International Journal of Biochemistry \& Cell Biology, 41, 40-59. http://dx.doi.org/10.1016/j.biocel.2008.06.010

[4] Pinsorrnsak, P. and Niempoog, S. (2012) The Efficacy of Curcuma Longa l Extract as an Adjuvant Therapy in Primary Knee Osteoarthritis, a Randomized Control Trial. Journal of the Medical Association of Thailand, 95, 51-58.

[5] Brien, S., Prescott, P., Coghlan, B., Bashir, N. and Lewith, G. (2008) Systematic Review of the Nutritional Supplement Perna canaliculus in the Treatment of Osteoarthritis. QJM: An International Journal of Medicine, 101, 167-179. http://dx.doi.org/10.1093/qjmed/hcm108

[6] Miller, M.R., Pearce, L. and Bettjeman, B.I. (2014) Detailed Distribution of Lipids in Greenshell Mussel (Perna canaliculus). Nutriments, 6, 1454-1474. http://dx.doi.org/10.3390/nu6041454

[7] Doggrel, S. (2011) Review Article: Lyprinol: Is It a Useful Anti-Inflammatory Agent? Evidence-Based Complementary and Alternative Medicine, 2011, Article ID: 307121. http://dx.doi.org/10.1093/ecam/nep030

[8] Kuptniratsaikul, V., Dajpratham, P., Taechaarpomkul, W., et al. (2014) Efficacy and Safety of Curcuma Domestica Extracts Compared with Ibuprofen in Patients with Knee Osteoarthritis; a Multicenter Study. Clinical Interventions in Aging, 9, 451-458. http://dx.doi.org/10.2147/CIA.S58535

[9] Coulson, S., Butt, H., Vecchio, P., Gramotnev, H. and Vitetta, L. (2013) Green Lipped Mussel Extract (Perna canaliculus) and Glucosamine Sulphate in Patients with Osteoarthritis: Therapeutic Efficacy and Effects on Gastrointestinal Microbiota Profiles. Inflammopharmacology, 21, 79-90. http://dx.doi.org/10.1007/s10787-012-0146-4

[10] Mickelborough, T.D., Sinex, J.A., Platt, D., Chapman, R.F. and Hirt, M. (2015) The Effect PCSO-524 a Patented Marine Oil Lipid and Omega-3 PUFA (Perna canaliculus) on Indirect Markers of Muscle Damage and Inflammation after Muscle Damaging Exercise in Untrained Men; a Randomized Placebo Controlled Trial. Journal of the International Society of Sports Nutrition, 12, 10. http://dx.doi.org/10.1186/s12970-015-0073-z 